\title{
Blocking Detection Based on Synoptic Filters
}

\author{
Bernd Schalge, Richard Blender, and Klaus Fraedrich \\ Meteorologisches Institut, KlimaCampus, University of Hamburg, Grindelberg 5, 20144 Hamburg, Germany \\ Correspondence should be addressed to Richard Blender, richard.blender@zmaw.de
}

Received 26 December 2010; Revised 1 March 2011; Accepted 21 March 2011

Academic Editor: Hann-Ming Henry Juang

Copyright () 2011 Bernd Schalge et al. This is an open access article distributed under the Creative Commons Attribution License, which permits unrestricted use, distribution, and reproduction in any medium, provided the original work is properly cited.

\begin{abstract}
The Tibaldi-Molteni blocking index is supplemented by additional filter criteria to eliminate cut-off lows and subsynoptic structures. We introduce three blocking filters and analyse their sensitivities: (i) a quantile filter requiring a minimum geopotential height anomaly to reject cut-off lows, (ii) an extent filter to extract scales above a minimum zonal width, and (iii) a persistence filter to extract events with a minimum duration. Practical filter application is analysed in two case studies and the blocking climatologies for the Northern and the Southern Hemisphere.
\end{abstract}

\section{Introduction}

Blocking is one of the most prominent flow patterns and has received attention during the last decades due to its influence on seasonal weather characteristics. The first qualitative criteria for the blocked atmospheric flow was introduced by Rex [1] in 1950 suggesting the atmospheric jet becomes split in two seperated parts with a considerable associated mass transport, the flow to be meridional at the splitting point and downstream, and the state to persist for at least ten days with a zonal width of at least 45 degrees. The original ideas formulated by Rex became the basis for several blocking indices. Most of the indices detect typical atmospheric patterns (e.g., [2, 3]). For shorter timeseries subjective criteria have been used (e.g., $[2,4]$ ). While the majority of the analyses consider the $500 \mathrm{hPa}$ geopotential height (e.g., $[5,6])$, Pelly and Hoskins [7], for example, detected blocking by a negative meridional potential temperature gradient on a potential vorticity surface (2 PVU level).

Despite the differences between the index definitions, they draw similar conclusions regarding the patterns of the Northern Hemisphere blocking climatology. However, the blocking frequency, which is the percentage of timesteps a certain longitude, is blocked, can be as low as 5\% [8], or higher than $20 \%$ [5]. The main reasons for this wide range of frequencies in automatic detection are modifications of the Rex criteria and the use of different parameters.
Due to its simplicity, the index of Tibaldi and Molteni (henceforth denoted as TM-Index) [5], which is based on the original idea of Lejenäs and Økland [9], has become a standard in automated blocking detection analyses. An important problem of the TM-Index is that it cannot effectively distinguish between blocking and cut-off low patterns, since both fulfill the TM-Index criteria. The modified TM-Index MTM [8] has a higher rejection rate for cut-off lows. Restrictions in the Rex criteria can be considered as implementations of independent filters which show synergetic effects if they are used in succession. The zonal TM-Index has been extended to latitudes by Scherrer et al. [10].

The main goal of the present study is to define filters and to determine their impacts on the detected blocking frequency using ERA-40 reanalysis data in a T106 resolution $\left(\approx 1.125^{\circ}\right)[11]$. The blocking analysis is based on the TMIndex of Tibaldi and Molteni [5] with an additional criterion to avoid gaps within blocked regions. A major aim of additional filters is to exclude nonsynoptic blocking flow anomalies and cut-off lows.

Three filters are applied:

(1) quantile filter, to ensure a positive anomaly in the geopotential height field in the blocked regions,

(2) extent filter, to remove structures below a zonal width, 


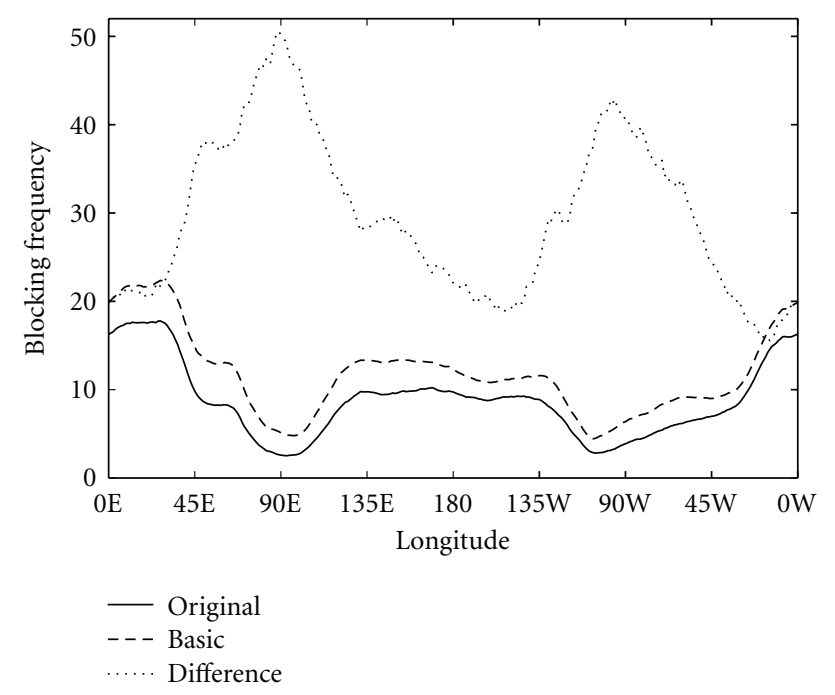

FIGURE 1: Northern Hemisphere blocking frequency: original TMIndex (solid), TM-Index for varying $\Delta$-ranges in (1) (dashed, basic setup), and relative increase (dotted, in \%).

(3) persistence filter, to remove structures below a duration threshold.

The filters are applied in two case studies and to the blocking climatologies for the Northern and the Southern Hemisphere.

The paper is outlined as follows. In Section 2, the blocking index including modifications is defined, and the data and the comparison methods are described. In Section 3, the results for the filters are given, as well as the results for the Southern Hemisphere. Section 4 contains the case study of blocking and cut-off low activity in February 1990. In Section 4, the results are summarised and discussed.

\section{Blocking Index and Filters}

In this section, the definition of the blocking index according to Tibaldi et al. [5] and the modifications are presented. The following filters are introduced:

(i) quantile filter, with a minimum threshold for the geopotential height,

(ii) extent filter, demanding a minimum zonal width, and

(iii) persistence filter, for a minimum life time.

The data used is the $500 \mathrm{hPa}$ geopotential height of the ERA-40 reanalysis dataset [11] (1958-2001) with a 6-hour time step and a spectral truncation of T106 $\left(\approx 1.125^{\circ}\right.$ on a Gaussian grid).

2.1. Blocking Index. The original criteria of Tibaldi et al. [5] for blocking at a longitude are

$$
\begin{gathered}
\text { GHGS }>0, \\
\text { GHGN }<-10 \frac{m}{{ }^{\circ} \text { lat }},
\end{gathered}
$$

with the geopotential height gradients in a northern and southern region

$$
\begin{aligned}
\mathrm{GHGN} & =\frac{Z\left(\phi_{N}\right)-Z\left(\phi_{0}\right)}{\phi_{N}-\phi_{0}}, \\
\mathrm{GHGS} & =\frac{Z\left(\phi_{0}\right)-Z\left(\phi_{S}\right)}{\phi_{0}-\phi_{S}} .
\end{aligned}
$$

In the present study, the TM-Index is modified by introducing different ranges for the gradients (this will be denoted as the basic setup in the following):

$$
\begin{aligned}
\phi_{N} & =78.75^{\circ}+\Delta^{\prime}, \\
\phi_{0} & =60^{\circ}+\Delta, \\
\phi_{S} & =41.25^{\circ}+\Delta^{\prime \prime}, \\
\Delta, \Delta^{\prime}, \Delta^{\prime \prime} & =\left[-3.75^{\circ}, \ldots, 3.75^{\circ}\right] .
\end{aligned}
$$

$Z$ is the geopotential height and $\phi$ denotes latitudes. Thus, the $500 \mathrm{hPa}$ geopotential height field is analysed for a pattern with a positive geopotential height gradient in the southern region and a strongly negative gradient in the northern region. Some studies neglect the criterion in the north for GHGN $<-10 \mathrm{~m} /{ }^{\circ}$ lat [12]. Here, the gradients GHGN and GHGS are computed for all possible combinations of latitudes in the ranges specified by the $\Delta$ intervals. A longitude is defined as blocked if for at least one case the criteria of (1) are satisfied. Since higher resolution data is used here compared to [5], the intervals for $\Delta$ include a larger number of latitudes and therefore more possible combinations.

The definition of a range for $\Delta$ leads to a larger number of detected blocking events. As an example, the possible latitudes for $\phi_{N}$ are $\left[82.43^{\circ}, 81.31^{\circ}, 80.19^{\circ}, 79.06^{\circ}, 77.94^{\circ}\right.$, $\left.76.82^{\circ}, 75.70^{\circ}\right]$ on a Gaussian grid. According to this definiton, it is possible to find blocking structures with a maximum between the centre and southern region. If, for example, only the southernmost latitude in the mid-latitude region has higher geopotential height than one of the more northern latitudes of the southern region, GHGS $>0$ and blocking will be detected. Without this modification, such a combination is not possible and blocking is not identified, because the high geopotential is too far south and the lower geopotential too far north, associated with a relatively small meridional extent of the block. The increase of the blocking frequency by the variable gradient ranges in the basic setup amounts to $20 \%$ in regions with high blocking frequency and to $50 \%$ in regions with low blocking activity compared to the original TM-Index (Figure 1).

Due to the restrictions to latitudinal gradients, the index defined here does not require a zonal extent of the blocking structure. In a small number of events, two seperated but close blocked longitudes can be identified which are in fact parts of one synoptic blocking event. Therefore, blocked regions with a gap less than $10^{\circ}$ are merged here. This occurs typically in the center of omega-shaped blocking where the southern criterion of (1) is not satisfied although a synoptic blocking is present. The merging has limited impact on 
the blocking frequency $(<5 \%)$ in the present form of the detection. However, if a zonal extent filter is applied, the merging can lead to a crucial exceedance of the threshold. This effect is evaluated in Section 3.

The blocking index defined in (1) together with the merging criterion still includes cut-off lows which should be excluded. Furthermore, the motivation for the identification of synoptic blocking events led to a comparative analysis of filter criteria applied in previous studies.

2.2. Filters. The analysis is based on the TM-Index (4) including the merging criterion, which is denoted as the reference setup. The main filters which are applied to obtain synoptic blocking are introduced in the following sections.

Quantile Filter. To eliminate cut-off lows, a positive geopotential height anomaly is demanded in the midlatitude region in addition to the TM-Index conditions (1)

$$
Z\left(\lambda, \phi_{0}\right)-Z_{Q}\left(\lambda, \phi_{0}\right)>0 .
$$

$Z_{Q}$ is the $Q$-quantile of the geopotential height for the particular latitude. For $Q=0.5$, for example, only longitudes with geopotential heights in the mid-latitudes higher than the median are allowed for blocking. Thus, the anomaly of the geopotential height in the mid-latitudes has to be positive compared to the respective $Q$ value for that latitude. This idea is motivated by a study of Dole and Gordon [6], who searched for persistent positive anomalies in the $500 \mathrm{hPa}$ geopotential height field.

With the criterion in (4) the number of cut-off lows is reduced (see Section 3). The Q-quantile is the threshold parameter to tune the stringency. However, this filter is not sufficient to demand synoptic scales of blocking highs.

Extent Filter. Hitherto, all longitudes are investigated for blocking seperately without regard to the zonal extent. The extent filter removes all blocking events with a zonal width below a prescribed threshold. The orginal criteria from Rex [1], which is based on subjective synoptic analysis, suggests a width of $45^{\circ}$. Since automatic detection routines based on the TM-Index yield distinctly smaller regions, a lower threshold is necessary to extract blocking events of similar extension. The reason is that the TM-Index extracts blocked longitudes inside the jet-split region rather than the whole blocked area [3]. Furthermore, smaller values for the extent filter ensure that not only the mature state of the block remains, but also parts of the onset and the decay phase. Note that the merging process introduced above inhibits spurious elimination of blocking events by the extent filter.

Since the zonal extent of a blocking event may vary during a life cycle, it is possible that the extent falls below the threshold temporarily, accompanied by a breakup into two temporal segments. If these segments are subject to a subsequent temporal filter requiring a minimum life time, this splitting leads to an underestimation of the blocking frequency. If such a temporal filter is not applied, counting of blocking frequencies is weakly influenced.

Persistence Filter. In this section, a filter is introduced to identify blocking events on synoptic time scales. This persistence filter removes blocked regions with a total life time below a prescribed number of timesteps. This filter acts in a Lagrangian frame since the blocking patterns are tracked. The identification in the tracking requires an overlap of at least one longitude for 6-hourly time steps. This algorithm is equivalent to the Spatial Criteria I from Barriopedro et al. [13] (the second criterion in this study is not used). If there is no overlap, the blocking event at the subsequent time step is considered as a new blocking. Note that this tracking condition is sufficient to detect individual blockings in high temporal resolutions like 6 hours.

There is a wide range of thresholds from 5 to 10 days for the persistence filter used in previous studies, with 10 days suggested by Rex, while more recent studies use a threshold of 5 days $[2,9]$. The durations of blocking events can vary substantially and can even exceed 30 days, depending on the large scale flow as well as on synoptic scale features of the circulation [14].

In summary, the application of all the filters with appropriate threshold values defined below, yields synoptic blocking events. It is important to note that the filters have to be applied in the sequence mentioned above.

2.3. Blocking Center Definition. For synoptic blocking events the center and the strength can be calculated. In this study the center is defined by the longitude within the block with the maximum GHGS, that is, where the difference of the geopotential heights between the midlatitudes and southern region is highest. The strength is defined by the maximum GHGS.

Although this definition might lead to fluctuations in the center for certain types of blocking (e.g., omega-shape patterns), there are two major advantages compared to other definitions: (i) the center cannot be at the edge of the blocking structure (as observed for the geopotential height maximum) and (ii) the center is identical to the location of the intensity maximum (and not simply the middle of the blocking as defined by geometric approaches). These aspects are considered in two case studies in Section 3.4.

\section{Results: Sensitivity and Climatology}

To obtain an overview on the impact of the individual filters, the blocking frequencies are analysed for each filter seperately (i.e., without the other filters). Blocking events are determined using the $500 \mathrm{hPa}$ geopotential height in ERA-40 data during 1953-2000. The analysis of the filter impacts is performed with Northern Hemispheric data, for the Southern Hemisphere a climatology is presented. Note that the filters have to be applied in the proper sequence given in Section 2.

3.1. Application to the Northern Hemisphere. In a first step, the impact of the merging criterion is assessed. Blocking is analysed in geopotential height data using the criteria in (1) with varying $\Delta$ and without any further filter. The blocking frequency is compared to the frequency obtained if all gaps smaller than $10^{\circ}$ between blocked regions are merged. It is evident from Figure 2 that the difference between 


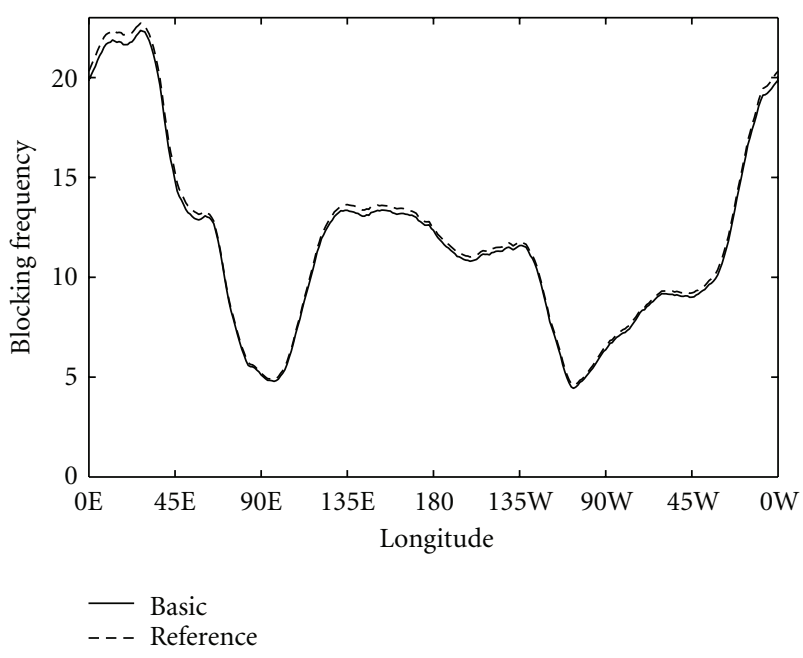

Figure 2: Northern Hemisphere blocking frequency: basic setup (solid, as Figure 1) and reference setup with closure of gaps smaller than $11.25^{\circ}$ (dashed).

these two methods is very small (approximately 3-5\%) and zonally homogenous. Obviously, the merging does not favor blocking frequencies in particular regions. Since merging of disconnected blocking regions is necessary for the identification of synoptic blockings which are subjected to the extent filter, this method is used as a reference setup in the analyses below.

All major features observed in previous blocking analyses are detected (compare e.g., $[6,7,9]$ ): High blocking frequencies over the eastern Atlantic and Europe, a small bulge for Ural-blocking at $60 \mathrm{E}$, high frequencies in a wide East-Asia/Pacific region (smaller than over Europe) and two distinct minima at 90E and 110W.

Quantile Filter. The quantile filter is designed to exclude cut-off lows. The impact of this filter depends strongly on the longitude (Figure 3(a)). The regions with the largest reduction in blocking frequencies are eastern Asia between $120 \mathrm{E}$ and 180 , with a peak at $145 \mathrm{E}$, and North America and the western Altantic from 50W to $90 \mathrm{~W}$. This shows that a substantial number of blockings which were detected in these regions in the reference setup are in fact cut-off lows. This effect is also observed in the case studies in Section 3.4. The shape of the blocking frequency is altered so that the Asia/West Pacific blocking sector is now less pronounced.

The choice of a threshold Q is a trade-off between elimination of cut-off lows and the erroneous elimination of synoptic blockings. With $Q=0.5$ (i.e., for positive anomalies with regard to the median), there are still some cut-off cases left especially in the mentioned sensitive areas. Using values higher than $Q=0.7$ can result in the exclusion of considerable amounts of synoptic blocking events. For example for $Q=0.8$ the total width of blocked regions cannot be higher than $72^{\circ}$ (out of $360^{\circ}$ ); this might be a too stringent exclusion for the simultaneous occurrence of two major blocking events. If this filter is used with $Q=0.5$, the excluded fraction is the frequency of cut-off lows. The regions that have the highest reduction in blocking frequency are also the regions with the highest cut-off activity (see Figure 3(b) for $Q$-values ranging fron 0.5 to 0.8 ).

Extent Filter. The extent filter shows regional differences, but with a much smaller magnitude than the quantile filter (Figure 4). The regions with the lowest reduction by the extent filter are at the longitude $0^{\circ}$ in the Atlantic/European sector and within $140 \mathrm{E}$ and $170 \mathrm{~W}$ in the Pacific sector. The largest reduction of blocking is observed at $120 \mathrm{~W}$. The extent filter smoothes the blocking frequency profile due to the elimination of small structures; a noticable example is the reduction of the secondary maximum in the vicinity of $120 \mathrm{~W}$. However, features like the bulge close to the Ural are not affected.

The extent filter is the reason why small gaps within blocked regions had to be closed in the merging process before; otherwise the extent filter would have discarded this blocking event since both subregions are not large enough to pass the filter. Thus, the preceeding merging process leads to an increase in the blocking frequency if an extent filter is used (compare Figure 5).

Persistence Filter. The persistence filter shows a similar impact as the extent filter since it also smoothes the profile in a comparable but weaker manner (Figure 6). Furthermore, the reduction of the blocking frequency is even more homogeneous with a single outstanding maximum between $120 \mathrm{~W}$ and $80 \mathrm{~W}$. For all temporal thresholds within 3 and 10 days, the climatological pattern of blocking is detected. For 10 days the overall blocking frequency decreases by more than $85 \%$.

3.2. Sensitivities of the Filters. The impacts of the persistence and the extent filter on the blocking frequency can be assessed by a gradual increase of the thresholds. Since the reduction of the blocking frequency by the persistence filter is homogeneous, it is possible to consider the zonal mean reduction. The reduction for thresholds between 1 and 10 days follows a linear increase up to seven days (Figure 7). Note that this pertains to the blocking frequency, while the persistence filter acts on the durations of the individual blocking events.

In a further analysis, the distribution of the total life time of blocking events is determined. The distribution is estimated by the difference in blocking frequency divided by the mean filter value. The life time analysis is restricted to 10 days so that events with a longer life time are disregarded (Figure 8). The numbers of the blocking events decrease with duration, but short-lived events contribute less to the blocking frequency.

A similar analysis is performed for the extent filter (Figure 7(b)). It is important to note that the thresholds are not equidistant (because of data resolution) since the width of the intervals vary between $5.625^{\circ}$ and $4.5^{\circ}$ in the histogram; this explains the nonmonotonous behavior. Here, it has to be remarked that there is a stronger dependence of the blocking frequency on the longitude.

The results for the reduction versus the corresponding thresholds are very similar for the extent and the persistence filters. However, the number of blocking events in the class 


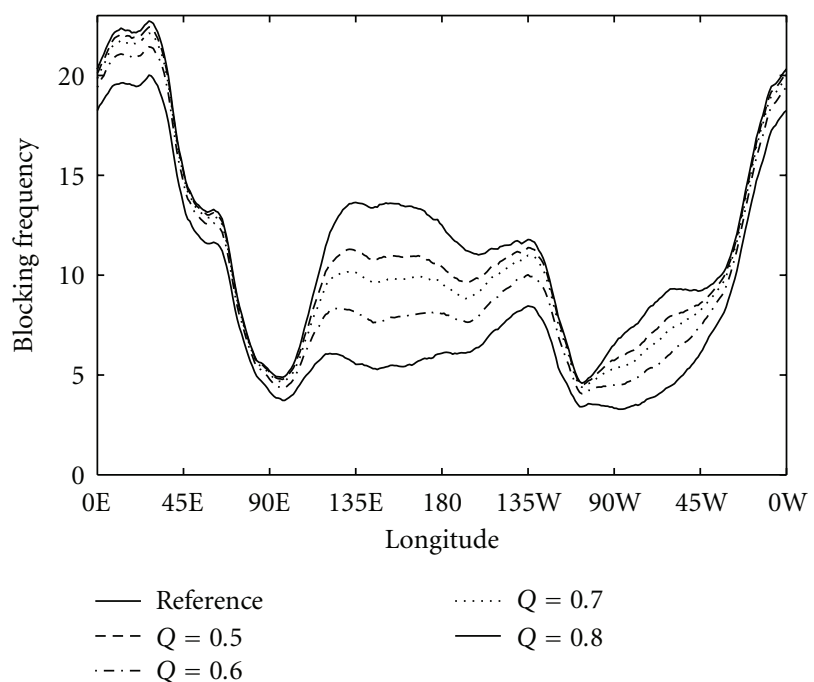

(a)

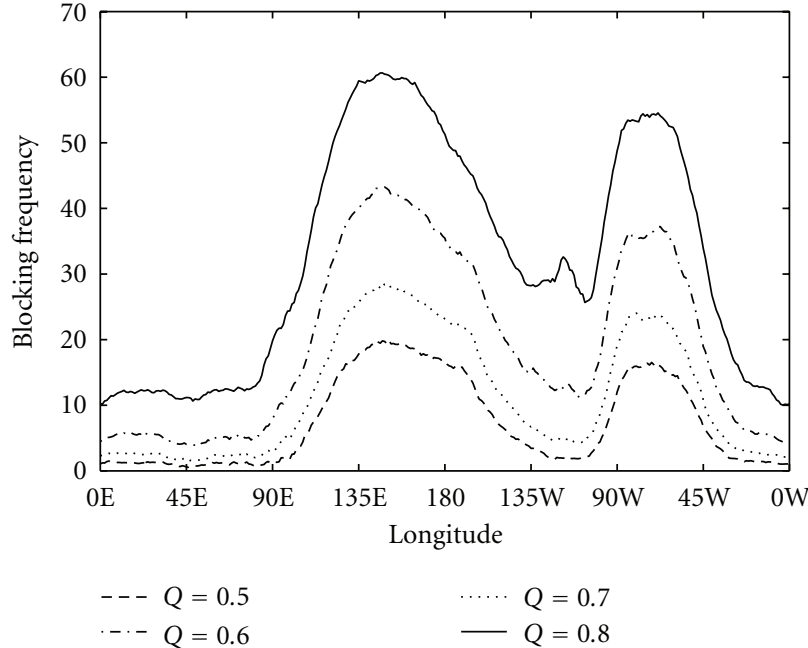

(b)

FIgURE 3: Quantile filter: Northern Hemisphere blocking frequency (a) reference setup and varying filter thresholds $Q$ and (b) relative changes.

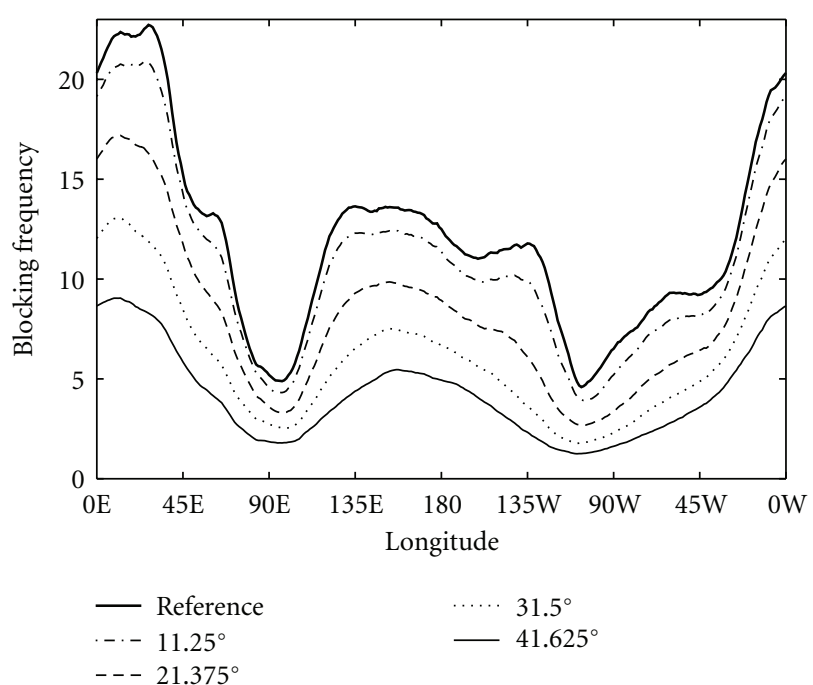

(a)

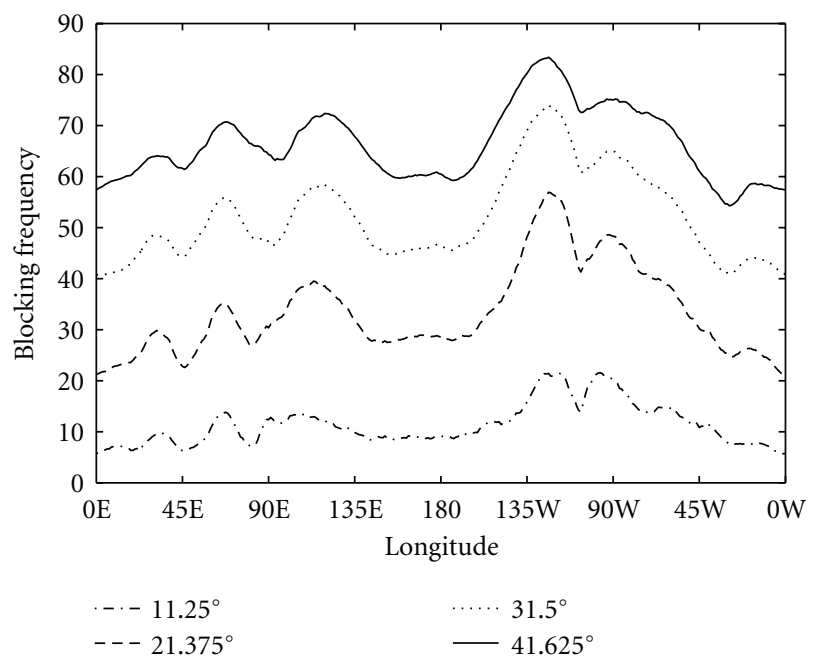

(b)

FIgURE 4: Extent filter: Northern Hemisphere blocking frequency (a) reference setup and varying filter thresholds, (b) relative changes.

with the smallest extension $\left(<5.625^{\circ}\right)$ is below the number in the subsequent class $\left(5.625^{\circ}-11.25^{\circ}\right)$. This indicates that the huge number of short-lived blocking events are not necessarily small.

A closely related hypothesis is that large structures are more persistent [2]. This is tested by an analysis of the persistence filter after the extent filter with a minimum extension of $21.375^{\circ}$ (Figure 9). The result indicates that even for structures larger than $21.375^{\circ}$ the reduction in blocking frequency is nearly linear up to 7 days as in Figure 7(a).
3.3. Southern Hemisphere. The following reasoning explains the increase for short temporal thresholds (see also Figure 13(c) in Section 3.4 below): (i) blocking structures with an extension slightly larger than the minimum extent which fall below the threshold for a short time and (ii) blocking structures which surpass the extent condition for a short time only. Both cases contribute to the numbers of short-lived events.

In summary, the combination of both filters, for example, a minimum extent of $21.375^{\circ}$ and 3 days in the persistence 


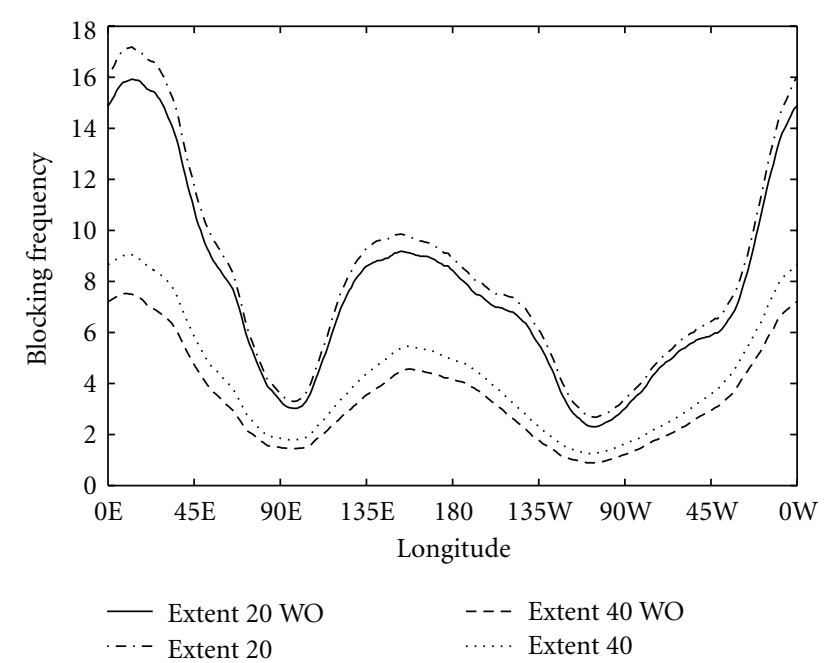

Figure 5: Extent filter and merging: Northern Hemisphere blocking frequency for the thresholds $21.375^{\circ}$ and $41.625^{\circ}$ compared to the case without merging (WO) as indicated.

filter extracts synoptic blocking events (with a prior quantile filter with $Q=0.7)$. Note that the threshold of three days permits the inclusion of transient block-like events. These events show all characteristics of a subjectively identified synoptic blocking:

(i) a distinct positive geopotential height anomaly,

(ii) a minimum extent of $2000 \mathrm{~km}$,

(iii) persistence of at least three days.

This conditions can be considered as a practical implemenation of the Rex criteria adapted for automatic blocking detection in gridded high resolution data.

Finally, in Figure 10, the blocking frequency for the recommended Northern Hemisphere threshold values is shown. The general distribution remains, however, a lot of details differ which have been discussed in the preceeding sensitivity studies.

The Southern Hemisphere ( $\mathrm{SH}$ ) blocking is far less frequent and not as persistent as its northern counterparts (e.g., [15-18]). The blocking frequency distribution shows only one large maximum in the Pacific region and a small one in the Atlantic, east of South America at $40 \mathrm{~W}$ (Figure 11). The comparison of the blocking frequencies in both hemispheres without filtering shows peaks above $20 \%$ in the Northern Hemisphere $(\mathrm{NH})$ while the corresponding $\mathrm{SH}$ values are barely above $6 \%$. Another prominent feature is a distinct minimum in the blocking frequency close to South America at 65W. Due to the different orography, the structure of blocking distribution on the $\mathrm{SH}$ is simpler compared to the $\mathrm{NH}$. There is only one region with a considerable amount of blocking in the Pacific between $150 \mathrm{E}$ and $80 \mathrm{~W}$ with a maximum at $150 \mathrm{~W}$.

If all the filters are used and set to the same parameters as in the $\mathrm{NH}, 21.375^{\circ}$ for the extent filter and 3 days for the persistence filter, the blocking frequency is significantly reduced compared to the reference TM-Index with a maximum below
$2 \%$. The maximum at $40 \mathrm{~W}$ vanishes, and the maximum in the Pacific region shifts eastward and is now located at $110 \mathrm{~W}$.

This result indicates that the thresholds applied in the $\mathrm{NH}$ have to be adapted for the SH to obtain statistically significant blocking frequencies. In Figure 11, the results found by three different combinations of extent and persistence filters are presented:

(i) extent filter threshold reduced to $15.75^{\circ}$, persistence filter 3 days,

(ii) extent filter $21.375^{\circ}$, persistence filter deactivated,

(iii) extent filter $15.75^{\circ}$, persistence filter deactivated.

For option (i), the difference compared to the NH settings is generally small, but the maximum at $40 \mathrm{~W}$ is distinctly reduced. Option (ii), however, shows some major differences. The overall pattern is more similar to the reference TM-Index than to the NH settings. The blocking frequency distribution is almost identical (on a lower level) except for the region east of South America, where the maximum in the blocking frequency disappears. The final option (iii) leads to a similar blocking frequency as (ii), but here as in (i), the maximum east of South America at $40 \mathrm{~W}$ is detected. For the SH, the quantile filter seems to be obsolete since the reduction in blocking frequency is negligible and zonally homogenous (Figure 11). This might indicate that SH cut-off lows and blockings do not occur in the same region. In summary, we conclude that the automated detection algorithm can be applied to the $\mathrm{SH}$ as well, but it needs to be adapted to the overall lower occurences of blocking events.

3.4. Case Studies. To demonstrate that the filters restrict the results of the TM-Index analysis to synoptic blocking events, two cases are considered explicitly here. The first example is a synoptic blocking event over Russia which has to pass all filters (otherwise, they would be too strict). The second example is a cut-off low detected by the reference TM-Index in the Pacific which has to be eliminated with the same settings.

Both cases occurred in February 1990. During this month, a blocking situation is observed over eastern Europe and Russia from Feb. 1318 UTC to Feb. 266 UTC and a cutoff event in the Pacific sector during the same time period from Feb. 2112 UTC to Feb. 2518 UTC. The dates given are the times of the first and the last detected blocked longitude of the respective event. In Figure 12, the geopotential height fields for both cases at their peak development are shown. This blocking event is exemplary, with a highest geopotential height around $65 \mathrm{~N}$ and 60E. 70 hours later the highest values are found at $57 \mathrm{~N}$ and $70 \mathrm{E}$; thus, the event is nearly stationary. The geopotential height values are about $5560 \mathrm{~m}$ for the former and $5640 \mathrm{~m}$ for the latter case. The Pacific cut-of-low has a very low geopotential height of $5000 \mathrm{~m}$ at its center at $48 \mathrm{~N}$ and $167 \mathrm{~W}$. This low, however, is embedded in a larger region with relatively high geopotential height. The development of this system is very fast and an example for rapid cyclogenesis, but it also decays relatively quickly, as it is detected for only 102 hours. 


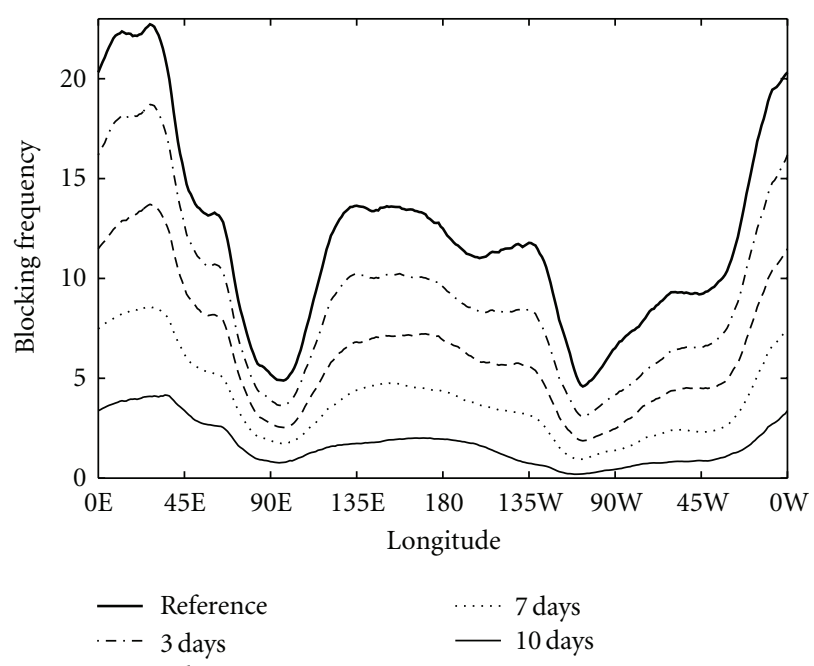

(a)

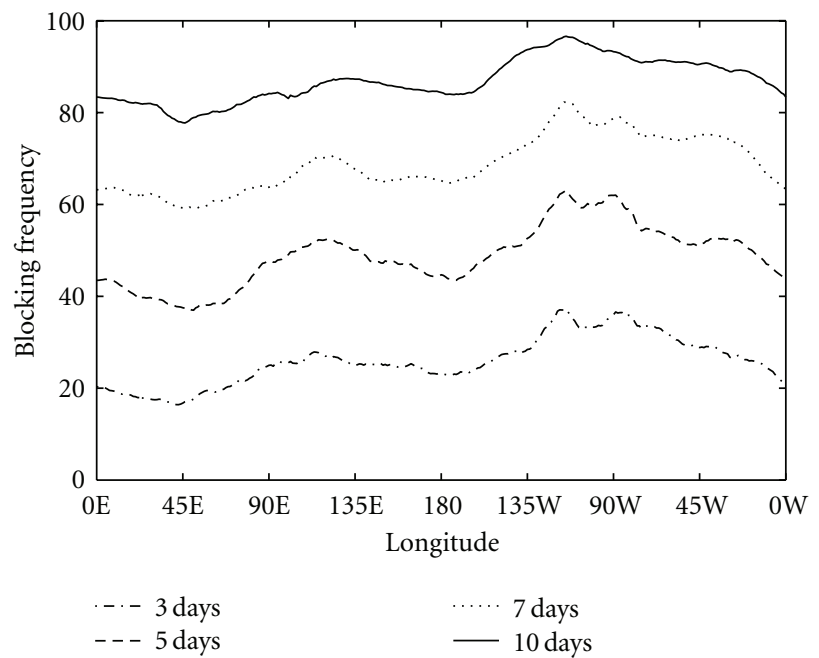

(b)

FIguRE 6: Persistence filter: Northern Hemisphere blocking frequency (a) reference setup and filter thresholds 3, 5, 7, and 10 days and (b) relative changes.

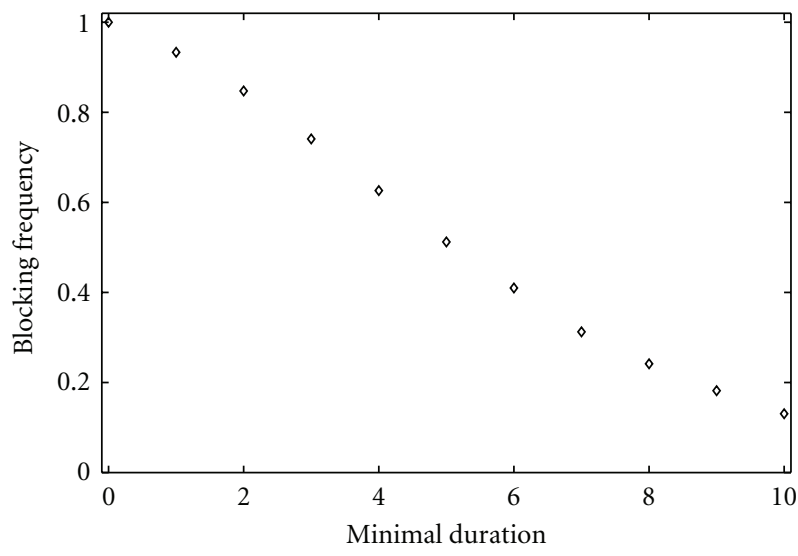

(a)

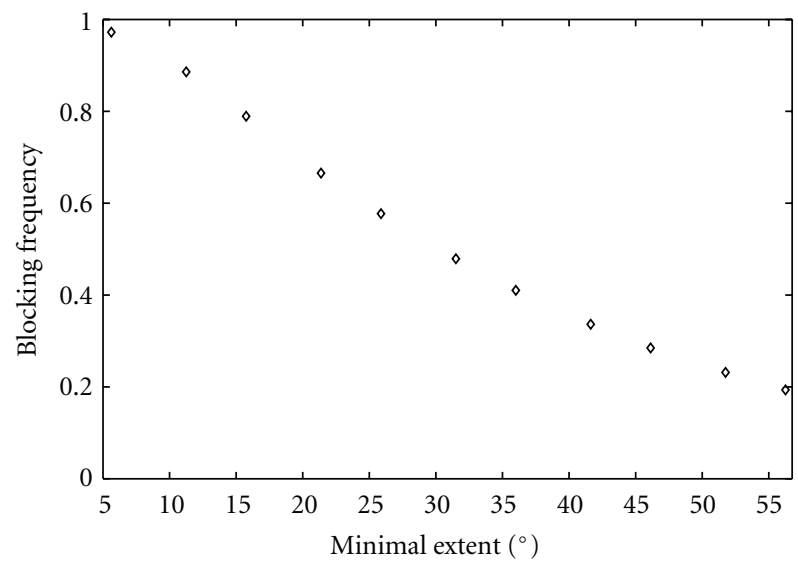

(b)

FIGURE 7: Reduction in zonal mean blocking frequency with increasing (a) persistence filter (without extent or quantile filters) and (b) extent filter (without time or quantile filters).

In the reference setup without filtering, both events are deteced as blocking event by the TMI. Since the cut-off low in the Pacific region is nested in a ridge with high geopotential relatively far north, the quantile filter is not sufficient to remove the structure, especially during the development and decay phases.

In Figure 13, a composite of Hovmöller diagrams for blocked longitudes during February 1990 for five different filter settings is shown: (a) without filter, (b) quantile filter, (c) extent filter, (d) persistence filter, and (e) all filters. It is evident that the combination of all filters removes the cut-off low completely, preserving the blocking event, which is detected for about five days from Feb. 1618 UTC to Feb. 2112 UTC.
This example demonstrates that cut-off lows are removed by a combination of the filters, while this cannot be achieved reliably with a single-filter method. The most powerful setup in this example is the combination of the extent and persistence filters. In this case, these two filters lead to the same result as all three filters, making the quantile filter obsolete. However, in general, cut-off-lows should be eliminated using all filters.

The combination of all three filters reduces the temporal extent of actual blocking events to the synoptically relevant segments, neglecting development and decay phases. In contrast to the quantile and extent filters, the persistence filter either removes blocking structures or preserves them completely. If this case study is performed exclusively with 


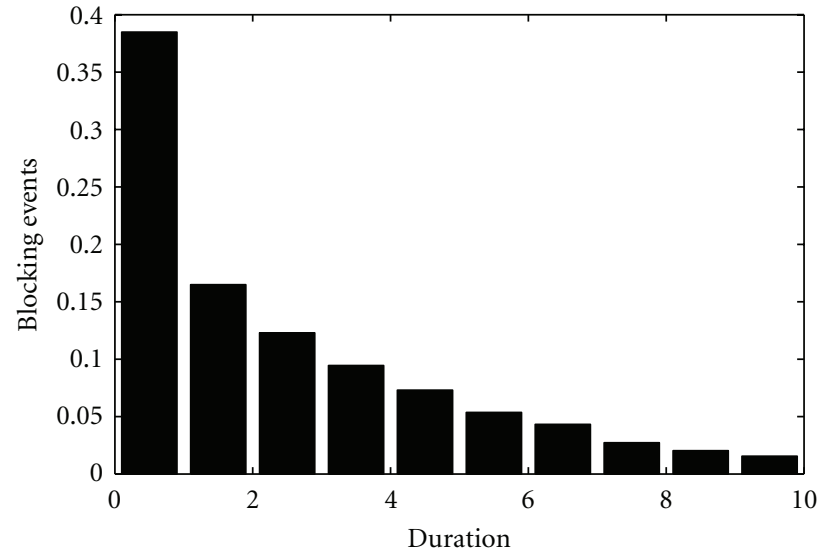

(a)

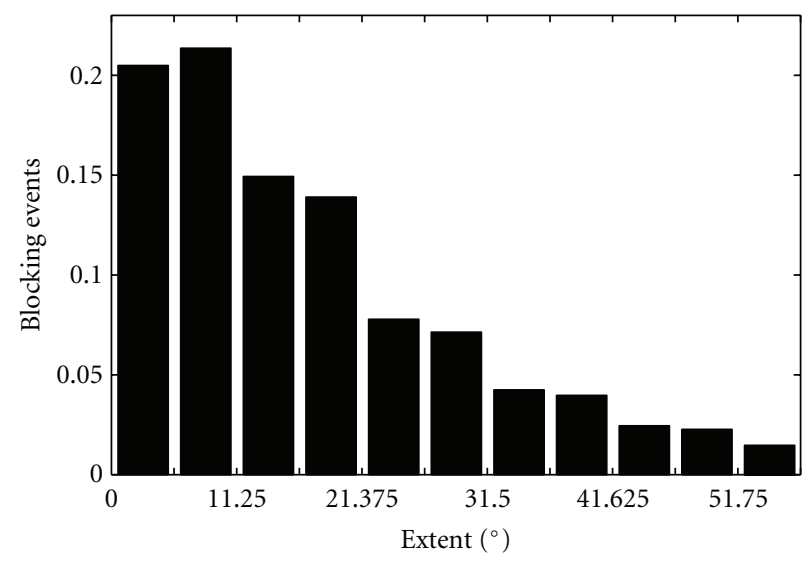

(b)

FIGURE 8: Histograms of the number of blocking events for increasing (a) persistence filter threshold and (b) extent filter threshold.

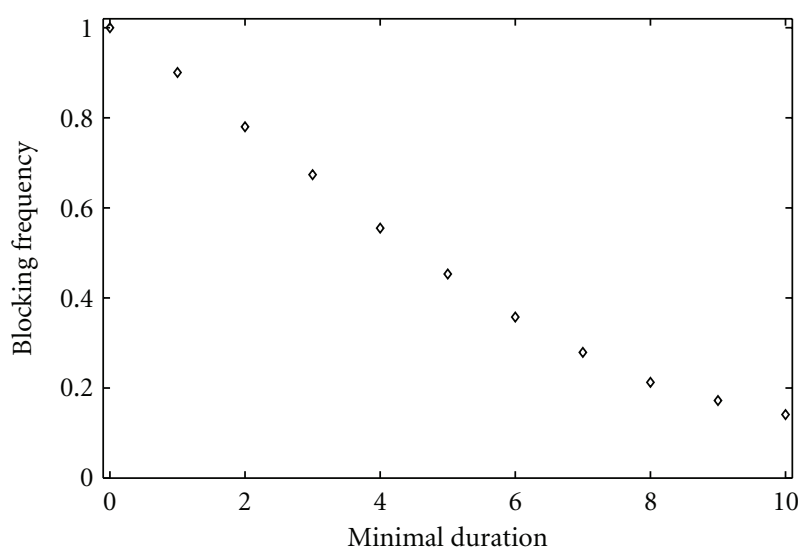

FIGURE 9: Reduction in zonal mean blocking frequency with increasing persistence filter (with extent filter width $21.375^{\circ}$, without quantile filters).

the persistence filter with a threshold of seven days, the result is the unabbreviated blocking event including development and decay phases, while all other structures are eliminated. Obviously, the persistence filter does not discard cut-off-lows if they exceed the temporal threshold.

\section{Summary and Discussion}

Some automatic blocking detection methods, for example the TM-Index suggested by Tibaldi et al. [5], are not suitable to find synoptic scale blocking, as they include a large number of smaller and short-lived structures. To compensate this deficiency, three filter criteria are introduced with the aim to restrict the outcomes to synoptic scale blocking events. The TM-Index is modified allowing a wider range of gradient measures and close blocked longitudes are merged. The impacts of the three filters are analysed independently.

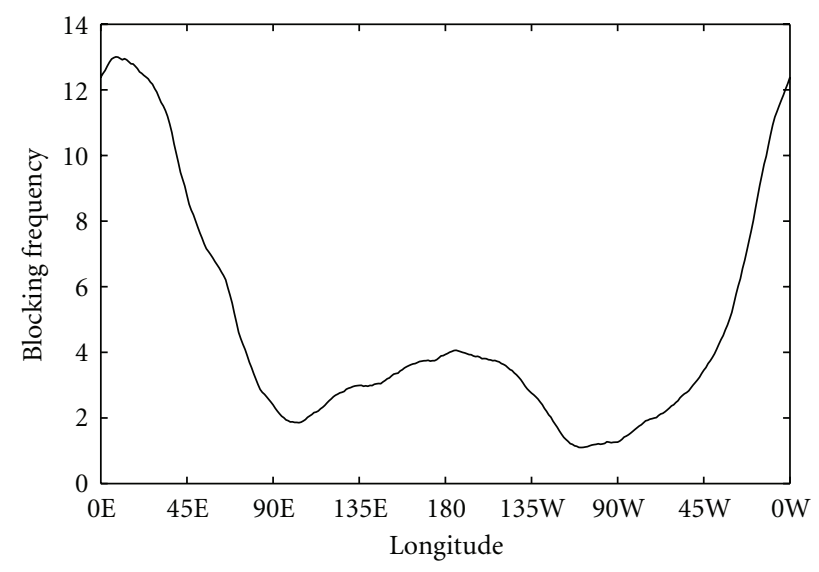

FIGURE 10: Northern Hemisphere blocking frequency for quantile ( $Q=0.7$, minimum width $21.375^{\circ}$, and 3 days duration).

In the final implementation, the filters cooperate and the thresholds in the individual filters are adjusted accordingly. For a successful implementation the sequence of the application is relevant. The three filters are implemented as follows.

(i) A quantile filter requires a minimum threshold for the geopotential height and is the basic method to eliminate cut-off lows. For a quantile $Q=0.5$ (median), which is roughly consistent with a threshold for positive anomalies, a complete elimination of cut-off lows is not observed. On the other hand, values higher than $Q=0.7$ should be avoided since this can lead to partial removal of synoptic blocking patterns. Therefore, the value $Q=0.7$ is recommended. This quantile filter shows a clear dependency on longitude.

(ii) The extent filter requires a minimum zonal extension of the blocking pattern to eliminate subsynoptic anomalies. The width of $45^{\circ}$ proposed by Rex [1] turns out to be not appropriate for automatic patterns which restrict to the core 


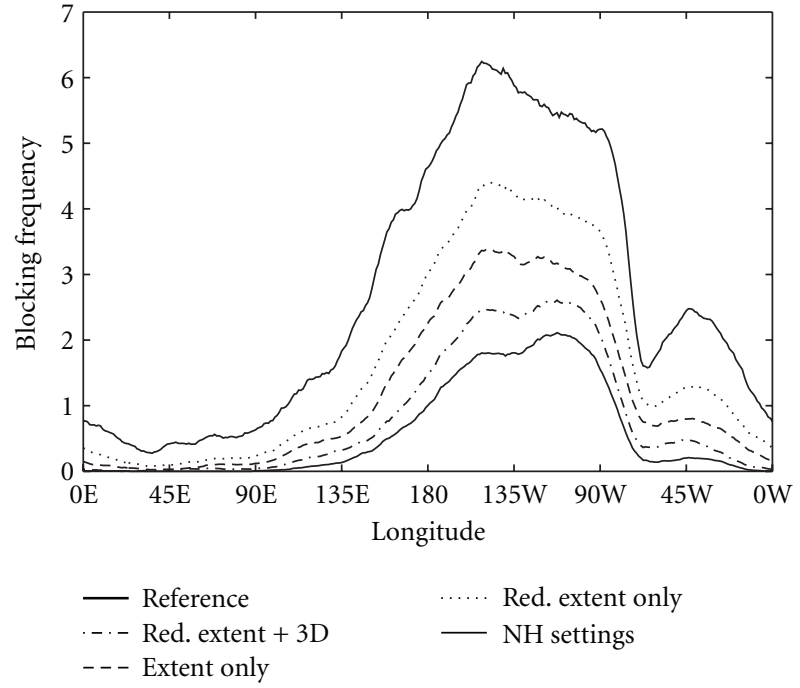

(a)

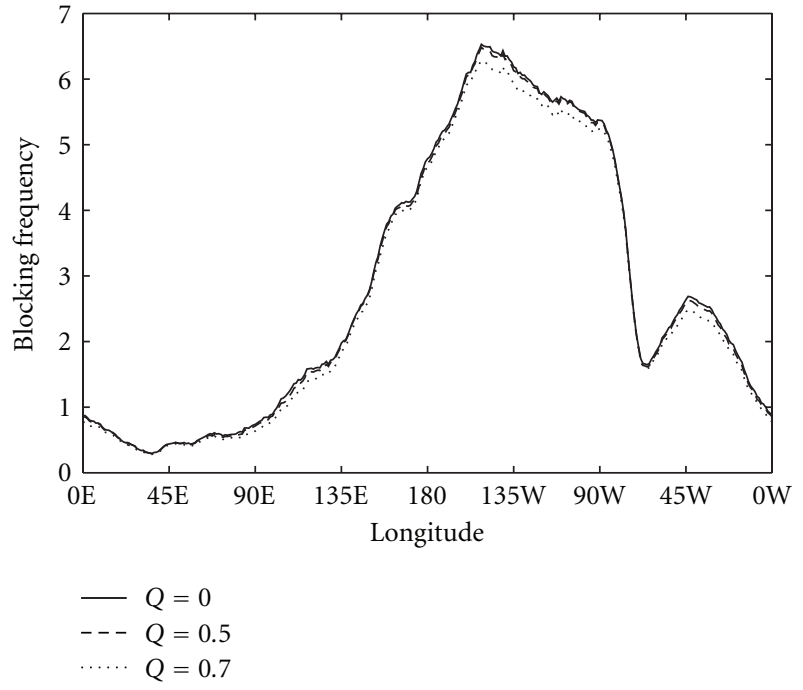

(b)

FIGURE 11: Southern Hemisphere blocking frequency: (a) for the reference setup (no filtering, solid, uppermost curve), NH settings (solid, lowermost curve), persistence filter disabled (dashed), extent filter reduced to $15.75^{\circ}$ (dash-dotted), persistence filter disabled and extent filter width $15.75^{\circ}$ (dotted) and (b) with quantile filters settings off (solid). $Q=0.5$ (dashed), and $Q=0.7$ (dotted).

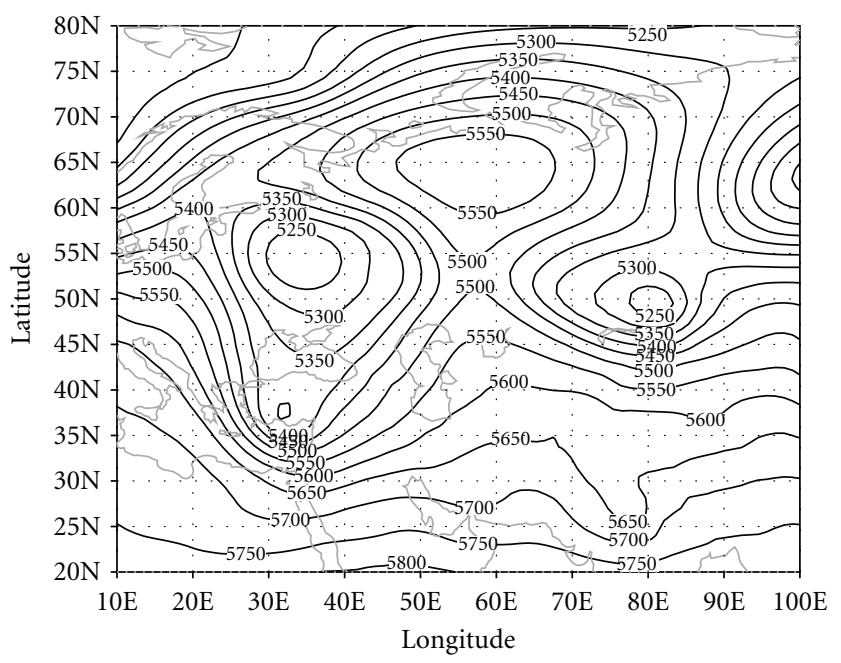

(a)

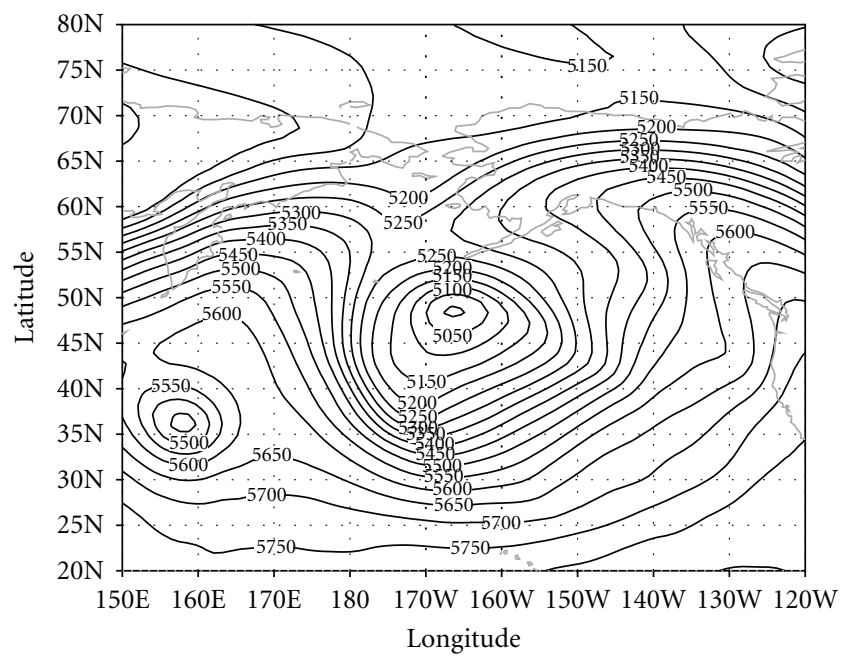

(b)

Figure 12: $500 \mathrm{hPa}$ Geopotential heights for (a) fully developed blocking Feb. 20th 00UTC 1990, (b) cut-off low Feb. 23rd 00UTC 1990.

of the event. In our analysis a width of $20^{\circ}$ appears as an optimum condition to extract synoptic scale anomalies.

(iii) The persistence filter demands a minimum life time for the blocking events which do not have to be stationary in our analysis. While Rex [1] suggests 10 days as a minimum condition, smaller thresholds have been used in other studies. In the present analysis, 3 days appear to be a sufficient minimum, in particular if this filter is combined with the quantile and the extent filters, since both discard growth and decay phases of blocking events.

The sensitivities of the extent and the persistence filters show a linear behaviour in wide threshold ranges. The impact of the persistence filter saturates at about 7 days, and the extent filter at $40^{\circ}-50^{\circ}$, a width which has been proposed by Rex [1]. Extent and persistence filter show a homogeneous blocking reduction which allows to estimate the number of events with certain spatial and temporal scales.

The analysis of the extent filter yields that only about $20 \%$ of all events are smaller than $5.625^{\circ}$, thus very small structures are rare. The persistence filter analysis shows that about $40 \%$ of all detected cases have a duration shorter than one day. This means that there is a large number of extended, but short-lived events. 


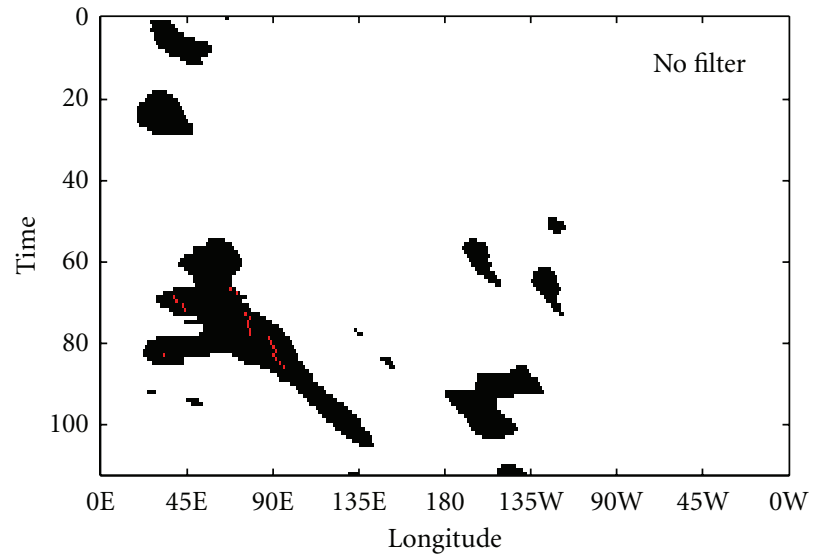

(a)

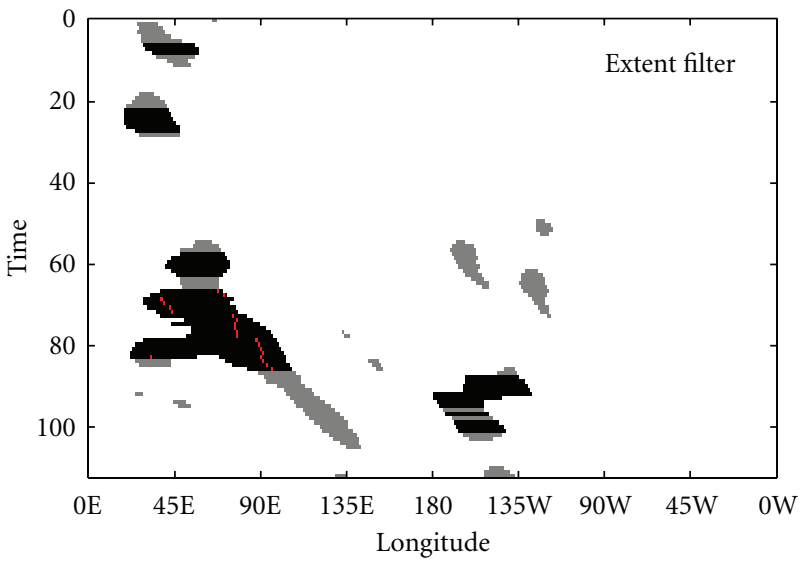

(c)

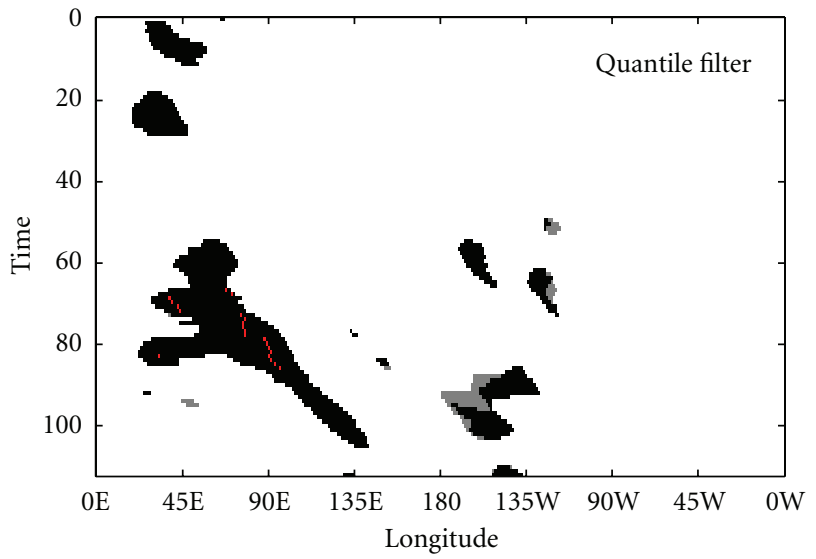

(b)

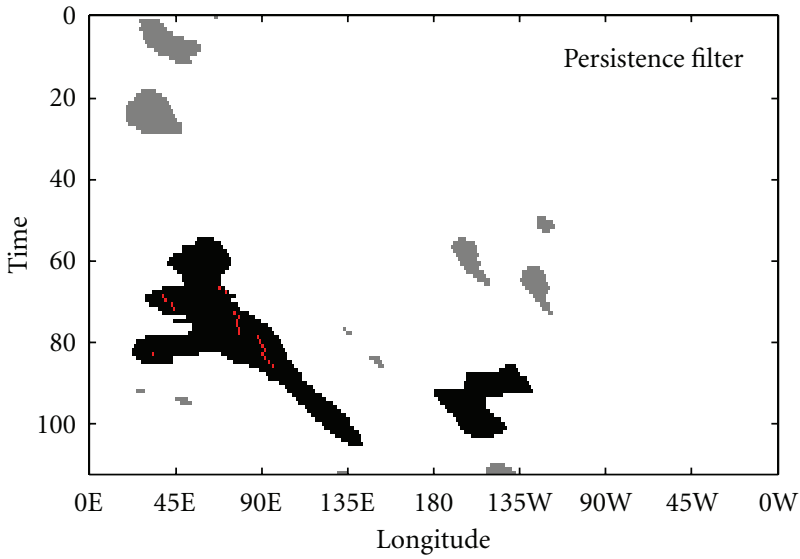

(d)

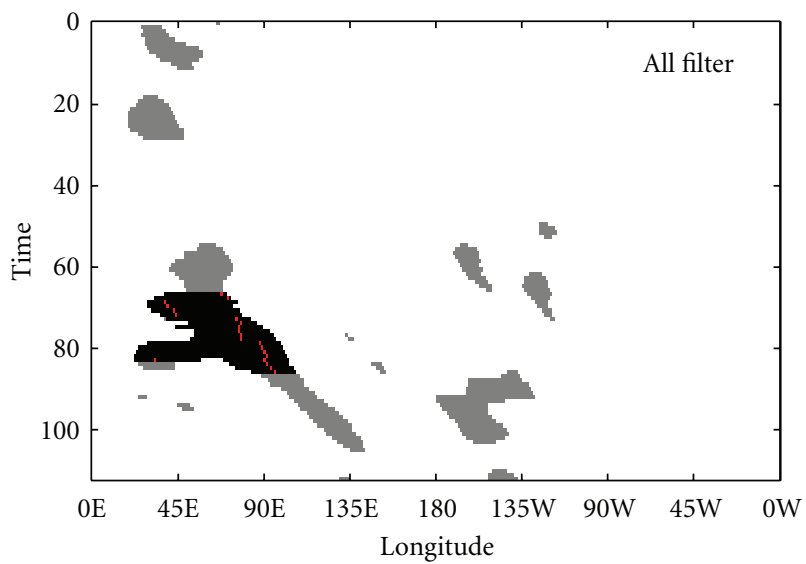

(e)

Figure 13: Hovmöller diagrams showing blocked longitudes for $6 \mathrm{~h}$ timescales and 112 time steps for February 1990 with blocked areas (black) and filtered areas (grey). The red marks indicate the center of the block (highest GHGS): (a) no filter, (b) quantile filter only, (c) extent filter only, (d) persistence filter only, and (e) all filters.

The case study of February 1990 demonstrates that applying all filters with the recommended threshold values does remove any structures that are not considered synopticscale blocking.

In the Southern Hemisphere, blocking events are far less frequent and are observed solely in the South Pacific.
Therefore, the extension of the present study to the $\mathrm{SH}$ requires a readjustment of the filter thresholds.

In summary, the combination of all filters with $Q=0.7$ for the quantile filter, $21.375^{\circ}$ for the extent filter and 3 days for the persistence filter applied to the TM-Index output extracts synoptic scale blocking events. 


\section{Acknowledgments}

The authors like to thank the two reviewers for their useful comments. B. Schalge acknowledges support from the KlimaCampus, and from the School of Integrated Climate System Sciences (SICSS).

\section{References}

[1] D. Rex, "Blocking action in the middle troposphere and itseffects on regional climate. I. An aerological study of blockingaction,” Tellus, vol. 2, no. 3, pp. 196-211, 1950.

[2] A. R. Lupo, "Climatological features of blocking anticyclones in the Northern Hemisphere," Tellus, Series A, vol. 47, no. 4, pp. 439-456, 1995.

[3] H. Lejenäs and R. A. Madden, "Traveling planetary-scale waves and blocking," Monthly Weather Review, vol. 120, no. 12, pp. 2821-2830, 1992.

[4] A. R. Lupo, R. J. Oglesby, and I. I. Mokhov, "Climatological features of blocking anticyclones: a study of Northern Hemisphere CCM1 model blocking events in present-day and double $\mathrm{CO}_{2}$ concentration atmospheres," Climate Dynamics, vol. 13, no. 3, pp. 181-195, 1997.

[5] S. Tibaldi, F. D'Andrea, E. Tosi, and E. Roeckner, "Climatology of Northern Hemisphere blocking in the ECHAM model," Climate Dynamics, vol. 13, no. 9, pp. 649-666, 1997.

[6] R. M. Dole and N. D. Gordon, "Persistent anomalies of the extratropical Northern Hemisphere wintertime circulation: geographical distribution and regional persistence characteristics," Monthly Weather Review, vol. 111, no. 8, pp. 1567-1586, 1983.

[7] J. L. Pelly and B. J. Hoskins, "A new perspective on blocking," Journal of the Atmospheric Sciences, vol. 60, no. 5, pp. 743-755, 2003.

[8] J. S. Watson and S. J. Colucci, "Evaluation of ensemble predictions of blocking in the NCEP global spectral model," Monthly Weather Review, vol. 130, no. 12, pp. 3008-3021, 2002.

[9] H. Lejenäs and H. Økland, "Characteristics of Northern Hemisphere blocking as determined from a long time series of observational data," Tellus, Series A, vol. 35, no. 5, pp. 350362, 1983.

[10] S. C. Scherrer, M. Croci-Maspoli, C. Schwierz, and C. Appenzeller, "Two-dimensional indices of atmospheric blocking and their statistical relationship with winter climate patterns in the Euro-Atlantic region," International Journal of Climatology, vol. 26, no. 2, pp. 233-249, 2006.

[11] S. M. Uppala, P. W. Kållberg, A. J. Simmons et al., "The ERA40 re-analysis," Quarterly Journal of the Royal Meteorological Society, vol. 131, no. 612, pp. 2961-3012, 2005.

[12] F. Kreienkamp, A. Spekat, and W. Enke, "Stationarity of atmosphericwaves and blocking over Europe-based on a reanalysisdataset and two climate scenarios," Theoretical andApplied Climatology, vol. 102, no. 1-2, pp. 205-212, 2010.

[13] D. Barriopedro, R. García-Herrera, A. R. Lupo, and E. Hernández, "A climatology of Northern Hemisphere blocking," Journal of Climate, vol. 19, no. 6, pp. 1042-1063, 2006.

[14] H. Athar and R. Lupo, "Scale analysis of blocking eventsfrom 2002 to 2004: a case study of an unusually persistentblocking event leading to a heat wave in the Gulf of Alaskaduring August 2004," Advances in Meteorology, vol. 2010, Article ID 610263, 2010.
[15] R. D. F. C. Marques and V. B. Rao, "Interannual variations of blockings in the Southern Hemisphere and their energetics," Journal of Geophysical Research D, vol. 105, no. 4, pp. 46254636, 2000.

[16] J. M. Wiedenmann, A. R. Lupo, I. I. Mokhov, and E. A. Tikhonova, "The climatology of blocking anticyclones for the Northern and Southern Hemispheres: block intensity as a diagnostic," Journal of Climate, vol. 15, no. 23, pp. 3459-3473, 2002.

[17] P. Berrisford, B. J. Hoskins, and E. Tyrlis, "Blocking and Rossby wave breaking on the dynamical tropopause in the Southern hemisphere," Journal of the Atmospheric Sciences, vol. 64, no. 8, pp. 2881-2898, 2007.

[18] J. A. Renwick, "Persistent positive anomalies in the Southern Hemisphere circulation," Monthly Weather Review, vol. 133, no. 4, pp. 977-988, 2005. 

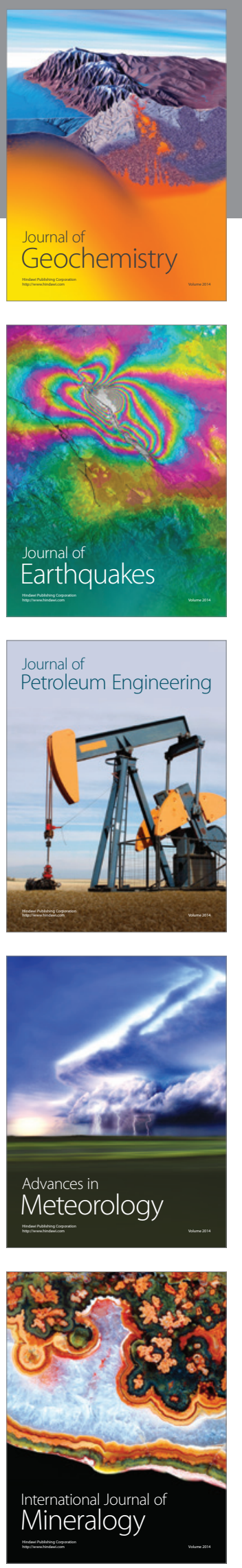
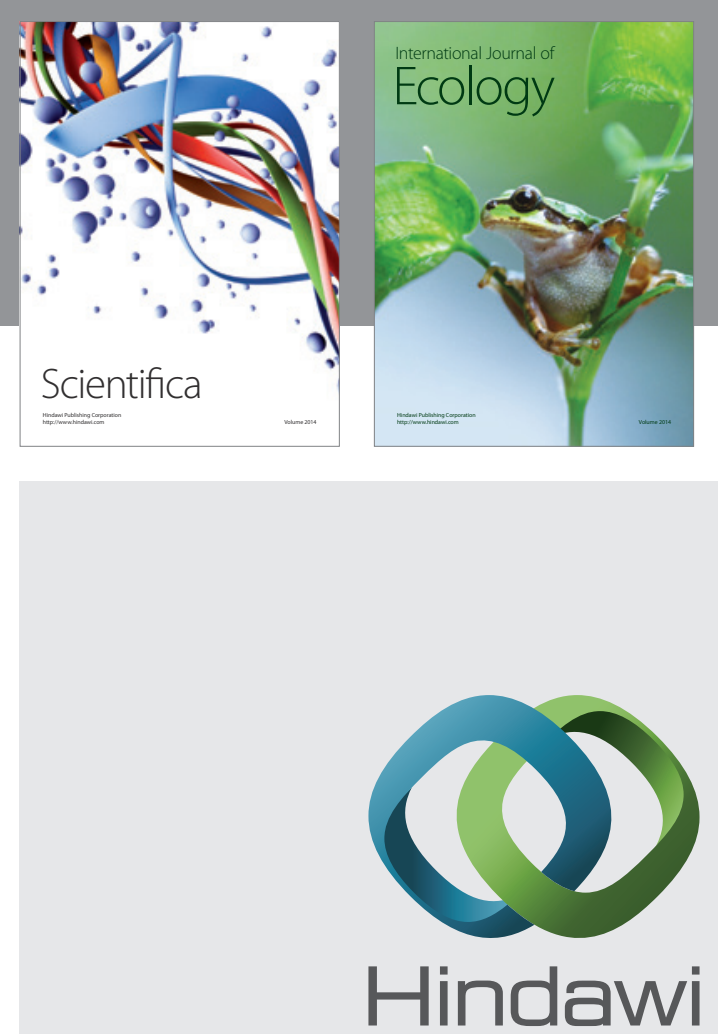

Submit your manuscripts at http://www.hindawi.com
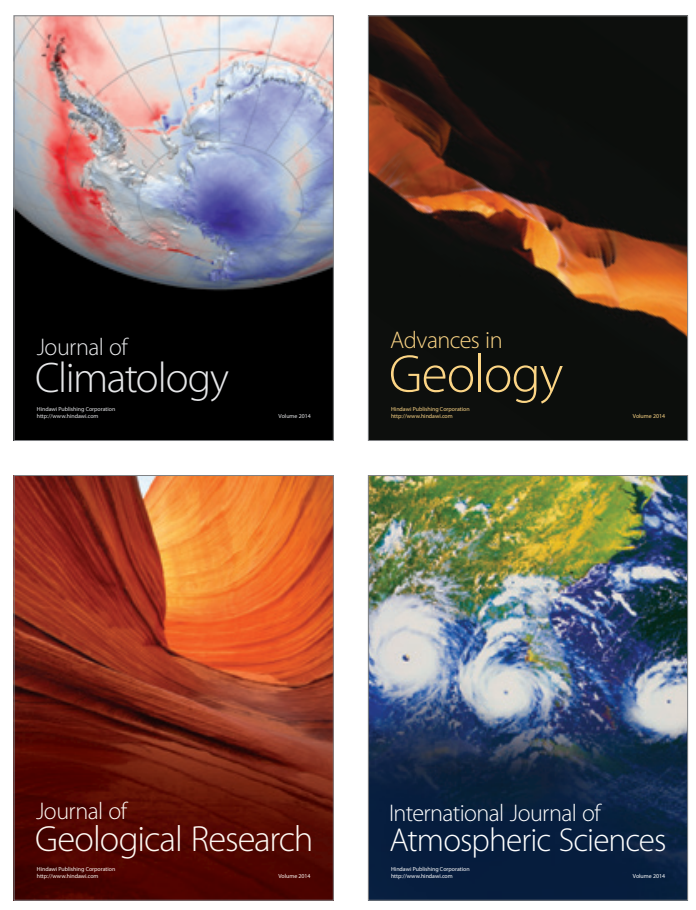
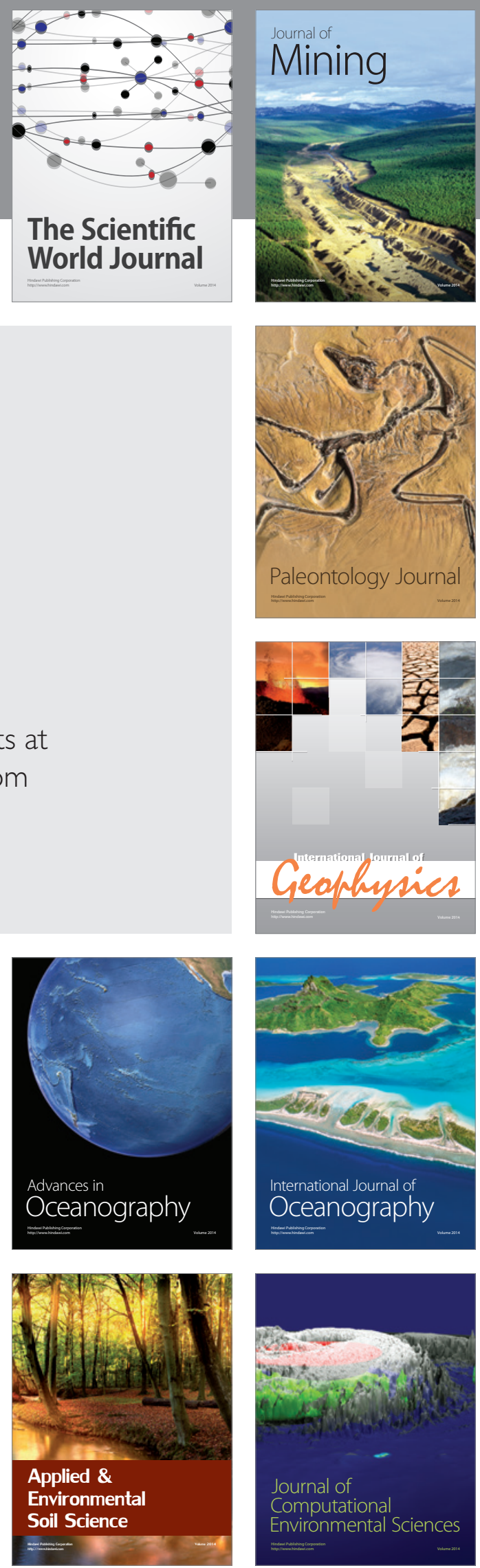